\title{
Japon : dépenses d'éducation et inégalités sociales
}

\section{Daisuke Sonoyama et Pierre-Louis Gauthier}

\section{(2) OpenEdition}

\section{Journals}

Édition électronique

URL : http://journals.openedition.org/ries/826

DOI : $10.4000 /$ ries.826

ISSN : 2261-4265

\section{Éditeur}

Centre international d'études pédagogiques

\section{Édition imprimée}

Date de publication : 1 septembre 2010

Pagination : $18-20$

ISBN : 978-2-85420-582-4

ISSN : 1254-4590

\section{Référence électronique}

Daisuke Sonoyama et Pierre-Louis Gauthier, « Japon : dépenses d'éducation et inégalités sociales », Revue internationale d'éducation de Sèvres [En ligne], 54 I septembre 2010, mis en ligne le 01 septembre 2013, consulté le 24 avril 2019. URL : http://journals.openedition.org/ries/826 ; DOI : 10.4000/ries.826

Ce document a été généré automatiquement le 24 avril 2019

(c) Tous droits réservés 


\title{
Japon : dépenses d'éducation et inégalités sociales
}

\author{
Daisuke Sonoyama et Pierre-Louis Gauthier
}

1 Le Japon a connu en 2009 un changement politique majeur avec l'arrivée au pouvoir du Parti démocrate, après cinquante ans de pouvoir de la droite. En dépit d'un programme politique ambitieux, la société japonaise est en train de perdre confiance, comme le montrent ${ }^{1}$ les recherches. En effet, le Japon se classe au cinquième rang de pauvreté relative de l'OCDE, derrière le Mexique, les États-Unis, la Turquie et l'Irlande. D'après la dernière enquête réalisée par le ministère de la Santé, une personne sur six atteint le seuil de pauvreté ( $7 \%$ pour la France).

2 Depuis l'enquête PISA, les chercheurs sont convaincus de l'importance du capital social et culturel qui, selon le « statut économique, social et culturel des parents », a creusé l'écart entre 2000 et $2006^{2}$. Pour la première fois depuis 1945 , le nouveau gouvernement a mis l'accent sur la nécessaire diminution des inégalités et la nécessité d'une société plus

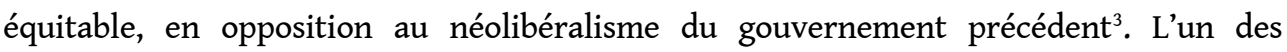
nouveaux engagements a été la mise en place d'une allocation de $200 \mathrm{E}$ par mois aux foyers en difficulté et la création de crèches.

\section{Le coût de l'éducation à la charge des parents}

3 L'article 26 de la Constitution japonaise affirme la gratuité de l'enseignement obligatoire pour les enfants âgés de 6 à 15 ans. Le Japon est l'un des pays de l'OCDE où, avec 4,1\% du PIB en 2006, la dépense de l'État pour l'éducation est la plus faible. Avec $22 \%$, la part relative des dépenses publiques et privées au titre des établissements d'enseignement, tous niveaux confondus, est la plus forte après la Corée ${ }^{4}$. Pour l'enseignement supérieur, la part de la dépense des ménages est plus importante : $51 \%$, à comparer aux $32 \%$ des dépenses publiques et aux $16 \%$ de la dépense d'autres entités privées.

En dépit de la loi sur la gratuité de l'enseignement obligatoire, les parents doivent fournir les matériaux nécessaires pour participer aux cours. D'après une enquête du ministère de 
l'Éducation et de la Science (MEXT, 2006), le montant de la dépense globale d'un élève inscrit dans un établissement public était en 2006 de $2715 €$ pour la première année de l'élémentaire, $3692 €$ pour le collège, et $4800 €$ pour le lycée.

5 La dépense totale pour un enfant, de l'école maternelle à l'université, pendant dix-neuf années passées dans un établissement public, atteint $62923 €$. La dépense totale pour un enfant scolarisé dans un établissement privé (sauf l'élémentaire, pour lequel il n'existe pas de statistiques), représente $120231 €$.

6 L'inscription à l'université est coûteuse. En moyenne, pour un étudiant vivant hors de la maison familiale et inscrit dans une université privée, les frais se montent à $76923 €$ pour quatre années de scolarité, alors qu'un étudiant vivant avec ses parents et fréquentant une université nationale ne coûte que $30769 €$.

\section{Quelques inégalités de la société japonaise}

7 D'après l'enquête, la part de l'éducation dans les dépenses dépasse $56 \%$ pour une famille au revenu compris entre $15385 €$ et $30769 €$. Elle n'est que de $23 \%$ pour une famille au revenu compris entre $69231 €$ et $76540 €$. Si le poids du coût de l'éducation est moindre pour les plus riches, le montant brut d'investissement reste néanmoins important ${ }^{5}$.

Depuis la décentralisation de 2005, les collectivités territoriales ont la charge de l'aide sociale aux enfants défavorisés. La part de l'aide pour les frais scolaires est passée de 6,6 \% en 1997 à 13,7\% en 2007. D'après l'enquête du MEXT, l'accroissement est lié à l'augmentation du chômage et des divorces. On peut noter également, dans les statistiques, l'augmentation du nombre de parents qui ne paient pas les frais de cantine ou celui des lycéens qui ne paient plus les frais de scolarité et risquent donc le décrochage.

9 Le système de bourses actuel est un prêt qui doit être remboursé par la suite, ce qui suppose un parcours sans erreur d'orientation. Il engendre une pression supplémentaire sur les élèves qui doivent réussir leur scolarité pour être sûrs d'être embauchés et de pouvoir rembourser.

10 Cet aspect sociologique et économique influence inégalitairement le taux de scolarisation à l'université. Selon les départements, il varie entre 32 et $71 \%$, alors qu'il est de $50 \%$ en moyenne nationale. L'écart social est important. Le taux de scolarisation dans une université privée varie de $22 \%$ pour une famille au revenu inférieur à $30769 €$, à $44 \%$ pour une famille au revenu supérieur à $76923 €$. Les universités se trouvant situées dans les grandes villes, les familles en zone rurale doivent payer, outre les frais de scolarité, le loyer et les frais de subsistance quotidienne.

11 Cette période de crise a vu les écarts de revenus se creuser. Depuis une décennie, le nombre des travailleurs ayant un contrat à durée déterminée a augmenté de $45 \%$. Cette progression reflète l'augmentation des petits emplois à temps partiel, dont le nombre a triplé. Le pourcentage de travailleurs de 30 à 59 ans ayant un revenu inférieur à $23077 €$, en-dessous du seuil de pauvreté pour les familles avec enfants, est passé de $8,9 \%$ à 13,3\%. 


\section{Un débat nouveau au Japon : la gratuité de l'enseignement}

12 Cela ne fait qu'élargir un peu plus le débat sur la gratuité de l'enseignement public et la responsabilité des parents (ou de la parentalité) en matière de réussite scolaire. Plusieurs recherches empiriques de sociologues, comme Kikkawa ${ }^{6}$, montrent que la clé de l'ascension sociale est déterminée par le niveau d'entrée au lycée. Jusqu'à aujourd'hui, le concours d'entrée et les frais de scolarité au lycée introduisent une hiérarchisation du lycée ouvrant soit vers les enseignements académiques, soit vers le monde professionnel. On peut constater que, selon le revenu des parents et l'accessibilité géographique, les jeunes ont une espérance scolaire différente dès la fin du collège. Le peuple japonais, lors de l'élection, a voté contre l'introduction du libre marché dans le service public d'éducation, qui accentuait l'inégalité économique et réduisait l'espérance ou les possibilités de parcours scolaire des jeunes.

En confirmant, pour la première fois, l'existence d'enfants pauvres, le nouveau gouvernement a permis de développer une politique en direction des couches sociales défavorisées, visant à les réinsérer dans la société en général et dans l'école en particulier, afin que tous les jeunes aient les mêmes chances d'accès au plus haut niveau de l'enseignement.

14 La lutte contre l'exclusion, basée des mesures sociales nouvelles, passe d'une politique financièrement limitée, favorisant l'exclusion, à une politique garantissant une protection sociale généralisée de l'enfance en matière d'éducation, de santé, de vie quotidienne. Malheureusement le nouveau gouvernement n'a pas trouvé les moyens financiers pour créer, par exemple, des institutions préscolaires destinées à accueillir les 25000 enfants sans place actuellement. Néanmoins, il a décidé de mettre en place, à partir du juin 2010, une allocation mensuelle de $100 €$ par enfant de 0 à 15 ans. De plus, les parents dont les enfants sont inscrits dans les lycées publics ou privés n'auront plus à payer les frais de scolarité à partir de l'année scolaire 2010.

Le Parti démocrate envisage également d'autres réformes administratives, notamment la décentralisation du pouvoir du ministère et du conseil central de l'Éducation pour donner plus d'autonomie à l'établissement scolaire. Il prévoit aussi le regroupement des différents services des ministères pour créer un nouveau ministère de l'Enfant, inspiré du modèle anglais, pour une politique plus cohérente.

\section{NOTES}

1. Yamada M. (2008). "Société d'inégalité dans les espérances ", éducation, famille, in Liberté, inégalité et individualité, CNRS, p. 185-198; Pelletier P. (2008). «Le mythe de l'égalité et de l'homogénéité sociales s'affaiblit », Questions internationales, n³0, p. 49-61 ; « Le Japon méconnu », Manière de voir, n 105, 2009; Kaneko (2008). «Égalité et inégalités dans la société japonaise 
d'après-guerre ", p. 265-280 et Garrigue-Testard et Chevallier (2008). «L'évolution des mentalités dans les années 1990 », p. 467-486 in Le Japon contemporain.

2. Fujita H. (2008). «Système politique et éducation. La réforme de l'enseignement en question », in Liberté, inégalité et individualité, CNRS, pp. 11-30.

3. Sonoyama D. (2006). «Une politique volontariste des savoirs et des compétences basée sur l'évaluation », RIE n 43, p. 47-55.

4. OCDE (2009). Regards sur l'éducation, Tableau B3.1 à B3.3. Part relative des dépenses publiques et privées au titre des établissements d'enseignement $(2000,2006)$.

5. Kodomo no Hinkon Hakusho (2009) (Livre Blanc sur la pauvreté des enfants). Akashishoten, p. 168.

6. Kikkawa T. (2009). Gakureki Bundan Shakai (La division du parcours scolaire), Chikuma Shinsho.

\section{INDEX}

Index géographique : Japon

Mots-clés : coût de l'éducation, inégalité sociale

\section{AUTEURS}

\section{DAISUKE SONOYAMA}

Daisuke Sonoyama est professeur adjoint à l'université Bunkyo d'Oïta (Japon). Sociologue de l'éducation, il travaille notamment sur la question des inégalités et sur des comparaisons France/ Japon, sujets sur lesquels il publié régulièrement.

\section{PIERRE-LOUIS GAUTHIER}

Pierre-Louis Gauthier est inspecteur d'académie (H). Il a été professeur d'histoire-géographie en école normale, inspecteur de l'éducation nationale, directeur d'école normale, directeur adjoint d'IUFM. Il a effectué des missions d'expertise des systèmes éducatifs pour la Commission européenne sur plusieurs continents. Membre du comité de rédaction de la RIE, pour laquelle il a coordonné plusieurs numéros, il collabore également à des revues catalanes, espagnoles et chinoises. 